\title{
Implantação da estratégia DOTS no controle da Tuberculose na Paraíba: entre o compromisso político e o envolvimento das equipes do programa saúde da família (1999-2004)
}

\author{
Implementation of the DOTS strategy in the control of TB in \\ Paraiba: between the political commitment and the involvement \\ of the teams of the family health program (1999-2004)
}

\author{
Lenilde Duarte de Sá ${ }^{1}$ \\ Marclineide Nóbrega de Andrade ${ }^{1}$ \\ Jordana de Almeida Nogueira ${ }^{1}$ \\ Tereza Cristina Scatena Villa ${ }^{2}$ \\ Tânia Maria Ribeiro Monteiro de Figueiredo ${ }^{1}$ \\ Rodrigo Pinheiro Fernandes de Queiroga ${ }^{1}$ \\ Maria Clemilde Mouta de Sousa ${ }^{1}$
}

${ }^{1}$ Universidade Federal da Paraíba. Rua da Falésia Condomínio Village Atlântico Sul, Praia do Seixas. 58045-550 João Pessoa PB.

lenilde_sa@yahoo.com.br.

${ }^{2}$ Escola de Enfermagem de Ribeirão Preto,

Universidade de São Paulo

\begin{abstract}
The scope of this paper is to analyze the implementation of the Directly Observed Treatment Short-Course (DOTS) strategy in the control of tuberculosis, from the standpoint of the coordinators of the Tuberculosis Control Program (TCP) in six priority municipalities in the state of Paraiba, Brazil. Semi-structured interviews were conducted with seven TCP coordinators. Five municipalities proved to be DOTS success points achieving a 90\% cure rate. Among the DOTS weak points in the political dimension, the following aspects were identified: lack of continuity of the TCP coordinator position; lack of preparedness of the local team; precarious technicaladministration structure and insufficiency of the laboratory network. In the operational dimension, the search for respiratory symptoms by the Family Health Teams is still low. Changes of an epidemiological, operational and political nature have been incorporated, though the implementation and guarantee of the sustainability of DOTS in the State depends on the way the health services are organized and on the manager's political commitment to support the strategy.
\end{abstract}

Key words Tuberculosis, Directly Observed Treatment, Family health, Healthcare systems
Resumo Foi analisada a implantação da estratégia Tratamento Diretamente Observado de Curta Duração (DOTS) no controle da tuberculose, sob a ótica dos coordenadores do Programa de Controle da Tuberculose (PCT), nos seis municipios prioritários da Paraíba. Foram realizadas entrevistas semiestruturadas com sete coordenadores de PCT. Fortalezas do DOTS: cinco municípios alcançaram a taxa de cura de 90\%. Entre as debilidades identificou-se, na dimensão política, a descontinuidade do cargo de coordenador de PCT, o despreparo da equipe local, a precariedade da estrutura técnico-administrativa e a insuficiência da rede laboratorial; na dimensão operacional ainda é baixa a incorporação da busca de sintomáticos respiratórios pelas Equipes de Saúde da Família. Ocorreram mudanças de cunho epidemiológico, operacional e político. a implantação e garantia da sustentabilidade do DOTS no Estado dependem do modo da organização dos serviços de saúde e o compromisso político do gestor no apoio a estratégia.

Palavras-chave Tuberculose, Terapia Diretamente Observada, Saúde da Família, Sistemas de Saúde 


\section{Introdução}

A tuberculose (TB) permanece como um dos problemas mundiais da saúde pública matando, pelo menos, 6 mil pessoas/ano no Brasil. Para o controle da doença a Organização Mundial de Saúde (OMS) propõe o Tratamento Diretamente Observado (DOTS) como estratégia para atingir $85 \%$ de cura, $70 \%$ de detecção de casos e reduzir o abandono ao tratamento em $5 \%{ }^{1}$.

O DOTS é constituído por 5 (cinco) componentes: detecção de casos por baciloscopia entre sintomáticos respiratórios que demandam os serviços gerais de saúde; tratamento padronizado de curta duração, diretamente observável e monitorado em sua evolução; fornecimento regular de drogas; sistema de registro e informação que assegure a avaliação do tratamento; compromisso do governo colocando o controle da TB como prioridade entre as políticas de saúde ${ }^{2}$. Em recentes fóruns nacionais e internacionais reconhece-se a importância do controle social e da organização dos serviços que, uma vez aliados aos cinco pilares, potencializam as ações para conter o avanço da TB.

O componente compromisso político relaciona-se à capacidade dos gestores em prover e articular recursos de informação e conhecimento, recursos organizativos, políticos e financeiros em função do tempo e de prioridades identificadas. Organizar serviços para a atenção à TB, implica em disponibilizar elementos estruturais (capacitação das equipes, insumos e rede laboratorial para realização de exames diagnóstico, provimento e abastecimento regular de medicamentos) na perspectiva de aperfeiçoar o processo de trabalho e o desenvolvimento das competências individuais e institucionais.

Considera-se, portanto, a qualidade da gestão em saúde como um importante elemento para o controle da TB, uma vez que, estando a estratégia DOTS inserida no âmbito da Atenção Primária à Saúde (APS), tende a acompanhar as adaptações e reformas contínuas do setor saúde ${ }^{3}$.

No Brasil a estratégia DOTS foi proposta no Plano Nacional de Controle da Tuberculose em $1998^{4}$, sendo então estabelecidas novas diretrizes de trabalho, com vistas à descentralização e horizontalização das ações de vigilância, prevenção e controle da TB, para o âmbito da atenção primária. Na primeira década do ano 2000 , observa-se expansão da cobertura dos serviços que utilizam a estratégia DOTS e o Brasil passou a pertencer ao grupo de países que possuem entre $50 \%$ a $90 \%$ dos serviços com a estratégia já implantada ${ }^{5}$.
Com a implantação da estratégia DOTS, avanços significativos podem ser comprovados na melhoria dos indicadores epidemiológicos relacionadas à TB no país. Em 2005 foram detectados $73,5 \%$ dos casos estimados, a taxa de cura alcançou 69,3\% - para todos os casos de TB - e $71,3 \%$ para a forma pulmonar bacilífera. No período de 1999 a 2005, evidenciou-se, também, uma redução de 5,7\% no coeficiente de incidência de casos de TB. Com a utilização do DOTS registra-se queda da taxa de abandono e o acesso garantido aos medicamentos, incluindo os casos de tuberculose multirresistente - (TBMR $)^{5}$.

Na Paraíba, o DOTS foi implantado em 1999, sendo inserido no Programa de Saúde da Família (PSF) de dez municípios selecionados por apresentarem, na época, população superior a 50 mil habitantes, maior carga bacilar e retaguarda laboratorial de referência ${ }^{6}$. Este período coincide com a expansão do PSF quando foi definida, no plano estadual de metas e diretriz para o ano $2000^{7}$ a ampliação do número de ESF e que tinha como um dos eixos a organização da atenção básica articulada às áreas consideradas prioritárias, dentre elas as ações de controle da TB.

Em 2001, o DOTS se expandiu para vinte municípios paraibanos mediante pactuação entre as instâncias estadual e municipal para a descentralização das ações do controle da $\mathrm{TB}^{8}$. A transferência de responsabilidade para o nível mais periférico do sistema vem redirecionando o fluxo de atendimento, deslocando gradativamente a atenção da Unidade de Referência especializada para as Unidades de Saúde da Família (USF). Neste sentido, desde o ano 2000, verifica-se nesse Estado uma estreita relação entre a ampliação das equipes do PSF e a extensão da estratégia DOTS.

Estas mudanças vêm provocando alterações nos indicadores epidemiológicos da TB. Entre 1999 e 2004, o percentual de cura aumentou de $67,8 \%$ para $92 \%$ e a taxa de abandono decresceu de 16,6 para $2 \%$, respectivamente ${ }^{6}$. No ano de 2005, 164 (73,54\%) dos 223 municípios da Paraíba contavam com o Programa de Controle da Tuberculose (PCT) implantado, correspondendo a uma cobertura populacional de aproximadamente $85 \%$. A cobertura populacional de PSF com DOTS é de $30 \%$ e a cobertura populacional da Paraíba com DOTS é 62,5\% ${ }^{6}$. O Tratamento Supervisionado (TS) vem sendo descentralizado para o PSF, sendo operacionalizado em $64 \mathrm{mu}$ nicípios e 204 USF.

Nos seis municípios considerados prioritários para o controle da TB a implantação do DOTS se deu no período de 1999 a 2003. Conse- 
qüente a essa implantação, tem-se observado que quatro deles alcançaram percentuais de cura elevados, em média superiores a $90 \%$, sendo dois da região metropolitana e dois do Sertão. A capital do Estado vem gradualmente apresentando melhora na taxa de cura variando de $44,4 \%$ em 1999 para $61,2 \%$ em $2003 \%$.

Considerando que os indicadores relacionados à TB estão estreitamente vinculados à política de reorganização do modelo de saúde iniciada no final da década de 1990 que elegeu o PSF como porta de entrada do Sistema Único de Saúde (SUS), este trabalho teve como objetivo analisar o compromisso político dos gestores na implantação da estratégia DOTS, sob a ótica dos coordenadores de PCT, nos seis municípios prioritários da Paraíba.

\section{Metodologia}

Este artigo resulta do projeto Situação da Implantação do DOTS para o controle da TB em algumas regiões do Brasil: histórico e peculiaridades de acordo com as características regionais. Para este estudo, foram selecionados seis (06) municípios prioritários para o controle da $\mathrm{TB}$ da $\mathrm{Pa}$ raíba, sendo a capital (João Pessoa), dois que integram a região metropolitana de João Pessoa (Bayeux e Santa Rita), um pertencente à região da Borborema (Campina Grande), dois localizados no sertão (Patos e Cajazeiras) e a capital ${ }^{4}$.

O estudo é de natureza qualitativa. Os dados foram coletados por meio de entrevista semiestruturada com o coordenador do PCT estadual e os coordenadores dos PCT dos municípios selecionados. As questões norteadoras centraramse no processo de implantação da estratégia DOTS, considerando as dimensões operacional e política.

Foram realizadas 07 (sete) entrevistas, complementadas com fontes secundárias, documentos oficiais e relatórios. Cada entrevistado foi identificado como el (coordenador estadual do PCT), e2 coordenador do PCT da capital), e3 e e4 (coordenadores dos PCT dos municípios da região metropolitana), e5 (coordenador do PCT do município da região da Borborema), e6 e e7 (coordenadores dos PCT dos municípios do Sertão). Como critério de inclusão justificou-se a atuação desses atores na implantação e desenvolvimento da estratégia DOTS nos municípios selecionados. Os dados foram analisados mediante a técnica de análise de conteúdo, modalidade temática ${ }^{10}$.
$\mathrm{Na}$ dimensão política considerou-se como categoria empírica o envolvimento dos atoreschave: gestores, coordenadores de PCT e equipe de saúde local. Na dimensão operacional considerou-se a transferência de responsabilidade das atividades técnicas e gerenciais do controle da TB para as ESF.

Nessas categorias foram buscados elementos significativos do comprometimento político de gestão local - negociação entre diferentes esferas de governo, recursos financeiros e humanos, incentivos, abastecimento de medicamentos, retaguarda laboratorial e treinamento. Na operacionalização privilegiou-se a distribuição da medicação, o TS e a busca de sintomáticos respiratórios (BSR).

Em atenção aos aspectos éticos e legais preconizados pela Resolução 196/96 do Conselho Nacional de Saúde, esta pesquisa foi aprovada pelo Comitê de Ética em Pesquisa da Escola de Enfermagem de Ribeirão Preto.

\section{Resultados}

A implantação e a sustentabilidade da estratégia DOTS, dentre outros fatores, depende do envolvimento de atores-chave no que tange a garantia do cuidado continuado ao doente de TB. Cabe aos gestores o compromisso político para que o controle da TB possa ser operacionalizado por outros atores envolvidos no processo, como os coordenadores de PCT locais e os profissionais que atuam principalmente no PSF. A respeito do apoio do gestor na implantação do DOTS, foi mencionado pelos coordenadores de PCT:

[...] nós tivemos também toda atenção do gestor estadual e gestores municipais. Eles estão cada dia mais conscientes de que o tratamento supervisionado é um tratamento que veio para ficar, e o Ministério da Saúde nessa gestão [...] priorizou a tuberculose [...].(e1).

[...] veio o pessoal do Ministério [MS] através da Secretaria de Saúde do Estado e o pessoal que, na época, fazia parte dessa gestão, aceitou. Achou interessante e a estratégia foi adotada no município $[\ldots]$ (...5).

Primeiro, ele [o gestor] concordou com a descentralização para as unidades de saúde, depois ele aceitou o processo da implantação da estratégia DOTS [...] (e7).

Constatou-se que, apesar do apoio dos gestores dos municípios, os critérios de repasse dos recursos financeiros não eram de conhecimento dos coordenadores do PCT, pois não possuíam 
recursos para o benefício dos doentes como auxílio deslocamento, cesta básica e café da manhã.

No inicio [...] eu não ficava por dentro das verbas [...]. Mas sempre que eu o procurava (o gestor) para liberação de vale transporte, a gente sempre era atendido. Na padaria ele (gestor) pagava o café dos pacientes (e2).

No tocante ao controle da TB, percebe-se que o apoio dos gestores dos municípios da PB foi importante nas mudanças de indicadores epidemiológicos da doença, fruto da descentralização de ações para o campo da atenção básica que, segundo os coordenadores de PCT, representou vontade política.

[...] com a descentralização para o município foi o compromisso total do município. O exemplo foi diminuir o indice de abandono, de transferência, de óbito... a partir da estratégia do DOTS (e6).

[...] a vontade política e a vontade da equipe. [...] o serviço de Tuberculose não é fácil [...] (e5).

Em relação aos municípios estudados, observa-se que a conformação do DOTS é singular em cada um deles, estando relacionada à forma de organização do sistema de saúde e ao compromisso político. Identificou-se nos municípios a convivência de diferentes graus de centralização e descentralização das atividades da estratégia DOTS. Neste sentido, nota-se que nos municípios prioritários a transferência de responsabilidades se deu de modo heterogêneo. As ações relacionadas ao sistema de informação e notificação foram transferidas para os PSF em apenas três dos seis municípios prioritários.

Tem uma funcionária que recebe a notificação, manda o boletim informativo no final do mês pra cada PSF mandar informação daquele paciente (e3).

De muitos problemas que cercam o controle da TB, os gestores devem priorizar a responsabilidade dos profissionais envolvidos com o trabalho na atenção básica. Segundo depoimentos dos coordenadores do PCT, alguns profissionais do PSF mostraram resistência em inserir ações de controle, sendo entendidas como mais uma atividade na rotina dos serviços ou em alterar o horário de chegada à USF.

É como falei, no início não foi fácil, porque a primeira [atitude] que a gente colocou era de fazer o café da manhã, teve dificuldade em questão do horário (e4).

Com relação à distribuição de medicamentos, os coordenadores de PCT informaram que esta ação foi descentralizada para as USF em cinco dos seis municípios prioritários.

A gente recebe trimestralmente da secretaria estadual de saúde, distribui trimestralmente para os municípios; no caso de Patos, a unidade de referência recebe trimestralmente, e mensalmente é distribuído para cada unidade que tem o paciente (e6).

Quanto à descentralização das atividades técnicas, tanto o TS quanto a BSR foram transferidas para as USF de quatro dos seis municípios. O TS foi a principal atividade descentralizada para as ESF em quatro municípios prioritários com população inferior a 130.000 habitantes. Os dois maiores municípios apresentam uma situação híbrida. Em um município, o TS é feito nas USF e na Unidade de Referência (UR). Na capital, o TS é feito nas UR e apenas em 34 das 180 equipes que integram a rede básica de saúde.

Observou-se que o exame de baciloscopia de escarro para diagnóstico permanece centralizado em todos os municípios prioritários, uma vez que não houve expansão da retaguarda laboratorial e nem tampouco esforço político dos gestores no sentido de reorganizar os serviços em função do número de laboratórios existentes. Os problemas de retaguarda laboratorial comprometem a produção de cuidado no SUS e o desafio de controlar a TB. Além desse desafio, um outro que se soma diz respeito ao preparo das equipes para a realização do TS.

[...] nós já capacitamos todos os ACS de Patos. [...] houve uma capacitação aprendizagem-ensino-serviço no controle da TB, e nós envolvemos a faculdade de enfermagem (e6).

Dentre as fortalezas identificadas na implantação do DOTS, destacam-se: o apoio do gestor em oferecer incentivos, a disposição da ESF em participar das capacitações e desenvolver estratégias para realizar o TS.

Os coordenadores de PCT apontam como fragilidades: dificuldade com os profissionais das equipes locais em aceitar mudanças nas rotinas para atender as necessidades especificas dos doentes em TS; a descontinuidade de cargos de coordenadores de PCT e pessoal técnico; significativa rotatividade dos profissionais do PSF; supervisão deficitária das atividades de controle da TB realizadas junto às ESF; falta de qualificação profissional para atuar no cuidado ao doente de TB; e deficiente retaguarda laboratorial.

No universo das fragilidades é importante fazer referência à implantação e sustentabilidade da estratégia DOTS na capital da Paraíba, um município de grande porte (população de 660.798 habitantes). Os resultados deste estudo indicam que há duas fases bem distintas. Entre 1999-2003, após a implantação da referida estratégia, observa-se um aumento do percentual de cura e se mantém a tendência de diminuição do percen- 
tual de abandono. Entre 1995-1998, antes da implantação do DOTS, observa-se uma queda tanto nos percentuais de cura como de abandono (Figura 1). No período 1999-2003, ocorreu um aumento do percentual de cura e uma redução nos percentuais de abandono. Sugere-se como explicação o fato de ter ocorrido, a partir de 1999, a implantação do DOTS com ênfase na atividade do TS (Figura 2).

\section{Discussão}

A introdução das atividades da estratégia DOTS no processo de trabalho em saúde dos municípios acarretou mudanças no mecanismo de gestão e, conseqüente, reformulação em sua condução, exigindo contratação de pessoal, disponibilização de recursos financeiros e incentivos para sua implantação. Neste sentido, a municipalização

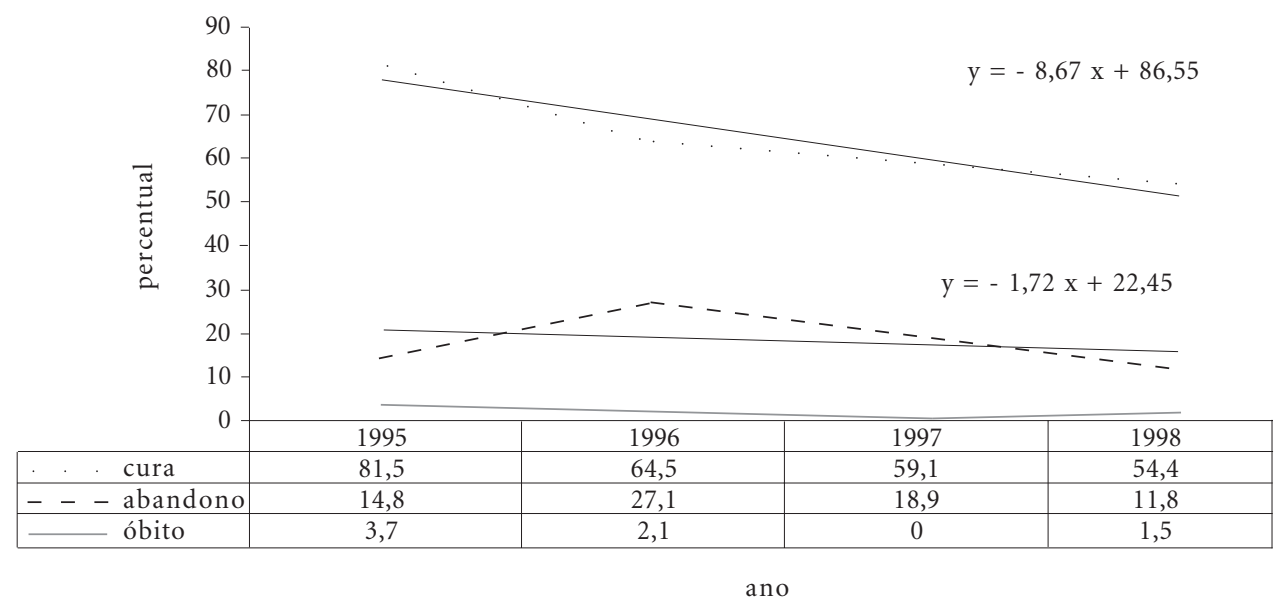

Figura 1. Percentual de cura, abandono e óbito, João Pessoa (PB), 1995-1998

Fonte: Implantação do DOTS em algumas regiões do Brasil. Histórico e peculiaridades regionais ${ }^{9}$

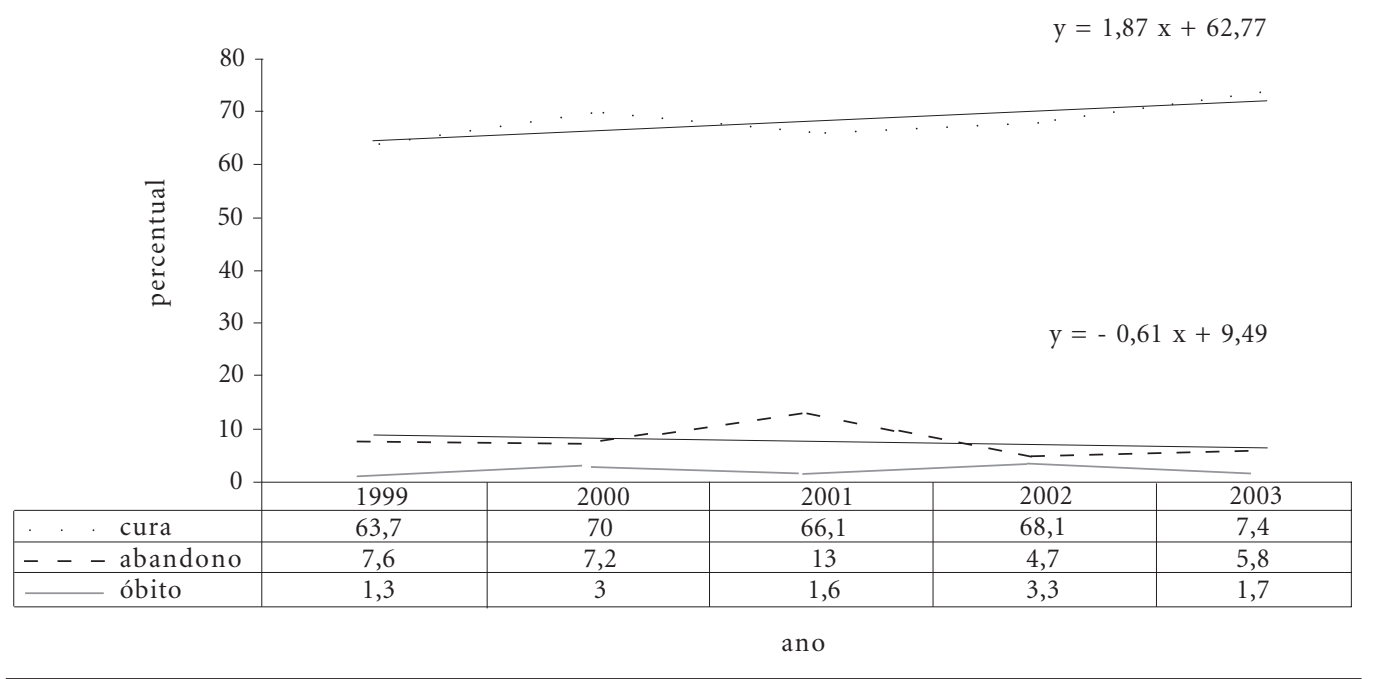

Figura 2. Percentual de cura, abandono e óbito, João Pessoa (PB), 1999-2003 
dos gastos em saúde tem como contrapartida um esforço crescente no que tange à responsabilidade do município na gestão dos recursos.

Didaticamente, podem-se identificar quatro grandes grupos de funções ou macro funções gestoras na saúde: formulação de políticas/planejamento; financiamento; coordenação, regulação, controle e avaliação; e prestação direta de serviços de saúde. Para que se exerçam efetivamente tais funções, é preciso que os gestores conheçam os problemas que permeiam o sistema local de saúde ${ }^{11}$. A atuação técnica do gestor do SUS como já se assinalou -, está permanentemente permeada por variáveis políticas, que podem ser definidas como um conjunto articulado de saberes e práticas de gestão necessárias para a implementação de políticas na área da saúde, exercidas de forma coerente com os princípios do SUS e da gestão participativa.

No relatório da $\mathrm{OMS}^{1}$ consta que o Ministério da Saúde, juntamente com outras autoridades dos níveis estadual e municipal deve trabalhar para fortalecer o controle da TB e reorganizar os serviços de APS para a implementação do DOTS. Isso é importante para assegurar melhores integração e coordenação das atividades, particularmente aquelas que incluem o PSF e o PACS.

Com o TS, as ESF tiveram de alterar a estrutura e o processo de trabalho de forma a atender a necessidade do doente de TB, uma vez que muitos teriam de tomar a medicação antes de partir para o trabalho, o que implicava abrir a USF antes do horário já pactuado, ou seja, mais cedo. A OMS ${ }^{12}$ considera também que para o efetivo controle da TB, é necessário que se organizem os serviços considerando a flexibilidade das equipes no acompanhamento do usuário, podendo a supervisão acontecer no domicílio ou mesmo no local de trabalho, além de poder ser realizada por alguém disposto, treinado, responsável, que o paciente aceite, e sob a responsabilidade de um profissional do serviço de saúde (PCT). Estratégias de cuidados devem ser criadas para potencializar o TS de modo a evitar que o usuário abandone o tratamento.

Em relação à importância do TS, em estudo desenvolvido sobre o abandono do tratamento da tuberculose em Cuiabá-MT, revela uma alta incidência de interrupção $(27,3 \%)$ e aponta o tratamento não supervisionado como uma das principais causas relacionadas ao problema. Para os autores do referido estudo, os resultados reforçam a importância da estratégia DOTS, visto que o TS evita que pacientes em tratamento inicial, por se considerarem assintomáticos, se percebam livres da doença e abandonem o tratamento. Os autores constaram ainda uma queda na incidência de abandono do tratamento da TB após a adoção do tratamento supervisionado pelo PCT local ${ }^{13}$.

No município paulista de Carapicuíba, no período de 2003 a 2005, a taxa de abandono do tratamento da TB dos casos em DOTS foi significativamente menor do que a dos casos em tratamento autoadministrado (TAA), com risco 2,3 menor de abandono, próximo da meta de $5 \%$ preconizada pela OMS. Os bons resultados estão relacionados à operacionalização do DOTS, uma vez que com essa estratégia há um contato mais íntimo, freqüente e humanizado entre o paciente de TB e a equipe de saúde, o que favorece a maior adesão ao esquema terapêutico e a conclusão favorável do tratamento ${ }^{14}$.

Observou-se também na Paraíba que as atividades de treinamento/capacitação das equipes de saúde local são desenvolvidas pelo nível estadual, ou seja, a atividade de qualificação dos trabalhadores da saúde ainda não foi descentralizada para o nível local. Reconhece-se que um dos maiores obstáculos do PSF está situado no setor de recursos humanos e qualificação dos profissionais ${ }^{14}$. Para o controle da TB, recomendamse treinamentos apropriados e contínuos, vistos como indispensáveis para a efetiva operacionalização da estratégia DOTS ${ }^{15}$. Portanto, o desafio é investir permanentemente em mecanismos de capacitação dos profissionais que atuam na estratégia Saúde da Família, seja na fase que antecede a implantação de uma proposta, seja no decorrer de suas atividades. A introdução de novas atividades no trabalho da equipe requer redefinição de papéis e mudanças na organização do processo de trabalho em saúde.

No que tange à organização do trabalho da Equipes de Saúde da Família em função da prioridade dada pelo Pacto pela Saúde-2006 para o controle efetivo de TB, esta se faz importante não apenas pelo ato de se promover um cuidado para devolver a capacidade de autonomia do usuário, mas, sobretudo, em impedir que aumente o número de casos de multirresistência (TBMR). O surgimento de casos dessa natureza, e que tem acentuado a gravidade do problema da tuberculose no contexto mundial, é relacionado a falhas do sistema de saúde, especialmente pela incapacidade de acompanhar o tratamento ${ }^{16}$.

Em se tratando da Paraíba, na capital, apesar implantação do DOTS ter sido iniciada em 1999, as atividades permaneceram centralizadas nas UR. Algumas ESF entregam a medicação, mas 
não fazem o acompanhamento da dose supervisionada. Assim, a dificuldade de implantação e sustentabilidade do DOTS pode ser explicada por ser um município de grande porte e apresentar um sistema de serviços de saúde que, historicamente, organizou a atenção à TB em unidades ambulatoriais. Desde 2005, as ações de controle da TB vêm sendo gradativamente descentralizadas para os PSF. A atividade de BSR está sendo implantada em cada ESF por meio do livro de registro para sintomático respiratório.

Admite-se que a forma de organização do sistema de saúde em municípios de grande porte favoreça a forma heterogênea de se gerenciar o cuidado, embora se reconheça que esses municípios tenham maior capacidade de captar recursos e de autonomia para gestão do sistema municipal de saúde. Por outro lado, verifica-se que as complexas formas de organização do sistema de saúde, promovem a execução das ações e serviços de forma heterogênea. No tocante à $T B$, as ações de controle podem ser impactadas pela dimensão político-gerencial, mediante a descontinuidade de cargos de gestor e coordenador do PCT, pelo acúmulo programas e serviços do coordenador. Enfim, o problema estaria relacionado à posição que a TB ocupa na agenda política de saúde do município ${ }^{17}$.

Constata-se que uma das resistências para implantação do TS na capital, tem sido a recusa por parte de alguns doentes para realizar o TS em PSF de seu território, optando pelo seguimento em PSF de outra área de abrangência. Essa ação do doente, que contraria a diretriz de regionalização traçada pelo SUS, precisa ser compreendida pelas equipes locais e coordenação do PCT, pela necessidade de preservação do anonimato devido ao estigma que, historicamente, acompanha a doença.

A situação da TB em Capina Grande - outro município paraibano considerado de grande porte - após a implantação da Estratégia DOTS, mostra que houve aumento do percentual de cura e diminuição da taxa de abandono. A expansão do número de ESF de 12, em 1998, para 46 em 2003, parece ter sido determinante para que a taxa de cura variasse de $75 \%$ em 1998 , para $83,2 \%$ em 2003. Com relação ao abandono no ano de 1998 a taxa era de $25 \%$, caindo para $6,9 \%$ em $2003^{9}$.
Dando ênfase à situação da TB dos outros municípios prioritários da Paraíba, observa-se que em Bayeux e Santa Rita, no período estudado, tiveram a situação epidemiológica da TB alterada após a implantação da estratégia DOTS. Nesses municípios, no período de 2000 a 2003 os percentuais de cura foram registrados acima de $85 \% \%$. No município de Cajazeiras, no mesmo intervalo de tempo, a taxa de óbito caiu de $11,5 \%$ para $0 \% 9$.

\section{Considerações finais}

Segundo os coordenadores de PCT, bem como outras fontes utilizadas para elaborar este estudo, conclui-se que a implantação e a garantia da sustentabilidade do DOTS no Estado da PB dependem da forma como se encontra organizado o serviço de saúde e o compromisso político do gestor no apoio à estratégia.

Observou-se que no período de 1998 a 2003, ocorreram mudanças positivas no quadro epidemiológico dos municípios prioritários, apesar de se reconhecer que há fragilidades que devam ser enfrentadas. A política de expansão do número de ESF que ocorreu pari passu à implantação da referida estratégia, parece ter sido determinante para o êxito que se observa no aumento de taxa de cura e diminuição de percentuais relativos ao óbito e ao abandono.

Constatou-se que as fragilidades da implantação e sustentabilidade da estratégia DOTS são reconhecidas pela descontinuidade política dos cargos de coordenador de PCT, o porte do município, alocação dos recursos financeiros condicionada à decisão do gestor, insuficiência de rede laboratorial para exame de baciloscopia e a falta de uma adequada política de qualificação de trabalhadores de saúde para atuar no controle da TB no SUS.

Os resultados encontrados justificam esforços dos gestores para investir na qualificação de recursos humanos envolvidos com o controle da TB e a promoção de políticas que priorizem a integração de saberes entre os setores de coordenação da atenção básica com as ESF em municípios prioritários para o controle da TB. 


\section{Colaboradores}

LM Sá participou da elaboração do projeto, coleta, análise dos dados e elaboração e revisão final do texto, MN Andrade da elaboração do projeto, coleta, análise dos dados e elaboração do texto, JA Nogueira da elaboração e revisão final do texto, TCS Villa da elaboração do projeto, elaboração do manuscrito e revisão final do texto, TMRM Figueiredo da elaboração do projeto e coleta de dados, RPF Queiroga da elaboração do projeto e coleta de dados e MCM Sousa da coleta de dados.

\section{Referências}

1. World Health Organization (WHO). Global tuberculosis control: surveillance, planning, financing. Geneva: WHO; 2005.

2. Organización Panamericana De La Salud (OPAS). Organización Mundial de la Salud (OMS). Reunión regional de directores nacionales de programas de control de la tuberculosis: informe final. Ecuador: OAS, OMS; 1997.

3. World Health Organization (WHO). An expanded DOTS framework for effective tuberculosis control: stop TB communicable disease. Geneva: WHO; 2002.

4. Brasil. Ministério da Saúde. Fundação Nacional de Saúde. Plano nacional de controle da tuberculose. Brasília: Ministério da Saúde; 1999.

5. Santos J. Resposta brasileira ao controle da tuberculose. Rev Saude Publica 2007; 41(1):89-93.

6. Lima DS. Plano de trabalho, em parceria com os gestores municipais dos municípios prioritários de: João Pessoa, Bayeux, Santa Rita, Campina Grande, Patos e Cajazeiras a ser cumprido em 2005-2006. João Pessoa: Secretaria de Estado da Saúde; 2005.

7. Veras CLSM, Rodrigues RM, Carvalho ALB. A saúde da família na Paraíba: do processo de implantação aos resultados. Divulg Saúde Deb 2000; 20:26-34

8. Paraíba. Secretaria de Estado da Saúde. Saúde da Família na Paraíba: Relatório de Atividades. João Pessoa; 2002.

9. Sá LD, Figueiredo TMRM, Lima DS, Andrade MN, Queiroga RP, Cardoso MAA, Villa TCS, RuffinoNetto A. A experiência da implantação da estratégia DOTS em seis municípios paraibanos. In: $\mathrm{Ru}-$ ffino-Netto A, Villa TCS, organizadores. Tuberculose: implantação do DOTS em algumas regiões do Brasil: histórico e peculiaridades regionais. Ribeirão Preto: FMRP, REDE TB-USP; 2006. p. 141-166.

10. Minayo MCS. O desafio do conhecimento: pesquisa qualitativa em saúde. $2^{\text {a }}$. ed. São Paulo: Hucitec, Rio de Janeiro: Abrasco; 1993.
11. Sousa MF, Fernandes AS, Araújo, CL, Fernandes MC. Gestão da Atenção Básica: redefinindo contexto e possibilidades. Divulg Saúde Debate 2000; 21:7-28.

12. World Health Organization (WHO). What is DOTS?: A guide to understanding the WHO-recommended TB Control Strategy Known as DOTS. Geneva: WHO; 1999.

13. Ferreira SMB, Silva AMC, Botelho C. Abandono do tratamento da tuberculose pulmonar em CuiabáMT - Brasil. Jornal Bras Pneumol 2005; 31(5):427435.

14. Vieira AA, Ribeiro SA. Abandono do tratamento de tuberculose utilizando-se as estratégias tratamento auto-administrado ou tratamento supervisionado no Programa Municipal de Carapicuíba, São Paulo, Brasil. J Bras Pneumol 2008; 34(3):159-166.

15. Monroe AA, Cardozo-Gonzales RI, Sassaki CM, Ruffino-Netto A, Villa TCS. Directly observed therapy and treatment adherence. J Bras Pneumol 2005; 31(1):91-92.

16. Vieira RCA, Fregona G, Palaci M, Dietze R, Maciel ELN. Perfil epidemiológico dos casos de tuberculose multirresistente do Espírito Santo. Rev Bras Epidemiol 2007; 10(1):56-65.

17. Villa TCS, Assis EG, MF Oliveira, Arcêncio RA, Gonzáles RIC, Palha PF. Cobertura do tratamento diretamente observado (DOTS) da Tuberculose no Estado de São Paulo (1998 a 2004). Rev Esc Enferm USP 2008; 42(1):98-104.

Artigo apresentado em 04/04/2008

Aprovado em 05/09/2008

Versão final apresentada em 30/09/2008 